\title{
Uma Análise de Confiabilidade em Pesquisas em Sistemas de Economia Colaborativa
}

\author{
Geanderson E. dos Santos, Raquel O. Prates \\ Departamento de Ciências da Computação (DCC) \\ Universidade Federal do Minas Gerais (UFMG) - Belo Horizonte - MG - Brasil \\ \{geanderson, rprates\}@dcc.ufmg.br
}

\begin{abstract}
This paper describes an investigation on the literature about trust in shared economy systems. To do so, we have applied a Systematic Literature Review (SLR) in this context. The SLR was applied to the main digital library databases in computer science - ACM, IEEE, and ScienceDirect. Sharing economy is a new kind of economy that allows users to share a human or physical resource that they are able to supply to other people who are interested in that resource. This new kind of economy has become very popular in the last few years, and its main platforms are: Uber and Airbnb. The main goal of this SLR was to investigate how trust is being considered in shared economy systems, analyzing which aspects are being considered when discussing trust, what research methods, either qualitative or quantitative, have been applied to measure trust on these types of platforms. Based on our analysis we have proposed a framework to describe trust indicators, as well as discussed methods being used to investigate trust and values being exchanged.
\end{abstract}

Resumo. Este artigo descreve uma investigação na literatura de como confiabilidade tem sido tratada em sistemas de economia colaborativa. Para isso realizamos uma Revisão Sistemática da Literatura (RSL) no tema. A RSL foi aplicada nas principais bases da computação, i.e., ACM, IEEE e ScienceDirect. Economia colaborativa se refere a um novo modelo econômico em que pessoas compartilham um recurso humano ou físico que tenham disponível com outros interessados. Esse novo modelo vem se popularizando muito nos últimos anos com plataformas como o Uber e Airbnb. O objetivo principal dessa RSL era verificar como a confiança era definida e percebida neste tipo de plataforma, e quais os métodos de pesquisa qualitativa elou quantitativa vem sendo usados para medir confiança nesse contexto específico. Como resultado de nossa investigação propomos um modelo de confiabilidade que descreve indicadores usados para analisar confiança, discutimos os métodos utilizados em trabalhos no tema, e os valores sendo trocados entre os envolvidos.

\section{Introdução}

A economia colaborativa, como foi originalmente definida por [Botsman and Rogers 2010], é baseada no excesso de bens de uma pessoa, uma massa crítica de participantes que queiram compartilhar esses bens, confiança entre desconhecidos, e o reconhecimento dos envolvidos no valor que este compartilhamento pode exercer na comunidade. Este novo movimento é caracterizado pelo compartilhamento de 
recursos, como por exemplo, quartos livres em residências, deslocamentos em veículos de outras pessoas, objetos e bens disponíveis para que outros usem, e uma variedade de serviços [Lampinen et al. 2015, Oh and Moon 2016]. Os maiores representantes deste novo tipo de economia são as plataformas Uber ${ }^{1}$ e Airbnb ${ }^{2}$ [Pargman et al. 2016].

Apesar do enorme sucesso de tais plataformas no contexto atual, muitas preocupações têm surgido em comunidades que usufruem desses serviços, sobretudo, após incidentes envolvendo abusos sexuais, roubos, danos morais, e outros problemas decorrentes do uso de aplicativos dessa natureza [Ert et al. 2016, Pick 2012]. Para evitar esses problemas, plataformas de economia colaborativa têm implementado soluções que ajudem usuários a desenvolverem algum tipo de confiança em outros participantes [Fradkin et al. 2015, Bilgihan et al. 2015, Nunes and Correia 2013]. De uma forma geral, esses mecanismos de confiança consistem em tentar construir algum tipo de reputação [Sánchez et al. 2016, Lumeau et al. 2015], tanto para aqueles que estão requisitando o serviço (demandantes), quanto para os que estão oferecendo o serviço (ofertantes).

Com base nesse cenário e no interesse de pesquisadores em entender os mecanismos de confiança que esses aplicativos desenvolvem [Sánchez et al. 2016, Lumeau et al. 2015, Lee et al. 2015, Fradkin et al. 2015], nosso objetivo neste trabalho é investigar o que significa confiança no contexto de economia colaborativa. Embora vários trabalhos explorem a questão de confiança em sistemas específicos [Ert et al. 2016, Nunes and Correia 2013, Ma et al. 2017], não encontramos nenhum trabalho que faça uma análise aprofundada de como a confiança tem sido definida e tratada no contexto de economia colaborativa como um todo.

Para conduzir esta investigação, uma Revisão Sistemática da Literatura (RSL) foi realizada que nos permitisse coletar dados e analisar a nossa questão de pesquisa $Q P$ : "Qual a definição de confiança no contexto de economia colaborativa?". Para conseguirmos melhor explorar aspectos desta definição, quebramos esta questão em três subquestões:

QP.1. "Quais são os indicadores de confiança encontrados na literatura?"

QP.2. "Quais são os tipos de métodos de pesquisa usados pela medir confiança?" e

QP.3. "Qual tipo de troca está sendo feita entre os participantes?”.

A partir da análise da RSL conseguimos identificar quais os indicadores de confiança têm sido estudados na literatura e quais as metodologias de pesquisa têm sido aplicadas para apreciar esses indicadores. Esses resultados permitiram a elaboração de uma proposta de um modelo de confiança que descreve para confiabilidade e os indicadores associados a cada um deles. Este modelo de confiança não é focado em nenhum sistema propriamente dito, e sua concepção engloba as principais dimensões da economia colaborativa. Além disso, essa pesquisa permitiu definir a confiança no contexto de economia colaborativa, mostrando uma evolução no conceito inicialmente proposto por seus idealizadores, sobretudo devido aos tipos de valores de troca que vem sendo utilizados nesses sistemas.

Na seção 2, apresentamos os trabalhos relacionados ao nosso. Logo depois, descrevemos a metodologia adotada, seguida pela apresentação e discussão dos resultados

\footnotetext{
${ }^{1}$ https://www.uber.com/pt-BR/

${ }^{2}$ https://www.airbnb.com.br
} 
obtidos. Por fim, tecemos nossas conclusões e indicamos trabalhos futuros de interesse.

\section{Trabalhos Relacionados}

A confiança na economia colaborativa é um tema que vem despertando o interesse de pesquisadores da computação e áreas afins (e.g., negócios e marketing). Essas pesquisas têm mostrado aspectos que podem ajudar no processo de desenvolvimento de confiança entre participantes, tanto em relação ao sistema, quanto na relação entre os usuários. Os trabalhos de [Sánchez et al. 2016, Lumeau et al. 2015, Lee et al. 2015, Fradkin et al. 2015] mostram que um dos mecanismos mais importantes para desenvolver confiança é a construção de reputação entre os usuários. Esses mecanismos de reputação estão sendo utilizados amplamente por plataformas peer-to-peer tradicionais, tal como o E-bay. $\mathrm{O}$ maior desafio mostrado por essas pesquisas é a migração desses mecanismos de reputação já estabelecidos em plataformas peer-to-peer para plataformas de economia colaborativa, considerando as diferenças entre os dois tipos de mercado e seus desafios particulares.

Outro mecanismo importante de confiança é a introdução de legislações que tornem os sistemas de economia colaborativa justos e aderentes às leis dos países que estão utilizando esse novo tipo de economia [Hartl et al. 2016, Pargman et al. 2016, Oh and Moon 2016]. A falta de legislações que regulem as plataformas têm feito com que a sociedade, de uma forma geral, não confie nos sistemas de economia colaborativa. Esses autores também destacam que a confiança é muito dependente da cultura de cada país, por isso, a introdução dessas plataformas em cada região é ainda mais complicada do que um sistema de software tradicional.

Como ferramenta de medição da confiança, os trabalhos de [Bilgihan et al. 2015, Nunes and Correia 2013] discutem a implementação e impacto de frameworks baseadas em modelos advindos de outras áreas do conhecimento, mas com o foco na confiança entre pessoas. Todavia, esses trabalhos não são focados na economia colaborativa em si, pois os autores consideram que os processos de confiança são imaturos em qualquer transação online existente entre usuários. Em um contexto diferente, o trabalho de [Dillahunt and Malone 2015] apresenta como a economia colaborativa pode ser introduzida em comunidades carentes para gerar algum tipo de renda para desempregados. Os autores notaram que os participantes conheciam a maioria das plataformas de economia colaborativa disponíveis no mercado (i.e., Uber, Airbnb, Lyft, NeighborGoods, entre outras), todavia, havia um receio dos participantes em utilizar essas plataformas justamente pela falta de confiança na economia colaborativa.

No trabalho de [Möhlmann 2016], os autores compararam a confiança nas plataformas de economia colaborativa com plataformas peer-to-peer de primeira geração (e.g., Ebay), assim como grandes plataformas de varejo online e não peer-to-peer (e.g., Walmart, Amazon, Zappos). O artigo conclui que a confiança em sistemas de economia colaborativa é ligeiramente menor do que a confiança em outras plataformas de mercado convencionais. Os achados chamam atenção para importância da confiança na economia colaborativa, sendo um dos principais desafios para o sucesso dos sistemas dessa natureza.

Em um tratamento específico de confiança focando em uma plataforma, a pesquisa de [Ert et al. 2016] apresenta um estudo que discute o papel de fotos pessoais no processo de confiança entre usuários do Airbnb. Os autores concluem que as fotos pessoais dos anfitriões do Airbnb são importantes para construir um sentimento de confiança 
entre os usuários. As fotos dos anfitriões obtiveram índices maiores de confiança do que as próprias revisões dos hóspedes, pois os autores também mostram que as revisões são, geralmente, consideradas boas ou muito boas para qualquer anfitrião, dificultando o processo de avaliação das melhores locações.

Em uma perspectiva similar, o trabalho de [Ma et al. 2017] discute como a quantidade de informações que um anfitrião do Airbnb disponibiliza em seu perfil influencia no aumento na confiança das pessoas que pretendem alugar aquele lugar. Aplicando métodos mistos de pesquisa, os autores usam um framework conhecido como Profile as Promise para medir o efeito das informações fornecidas nos perfis dos usuários. Eles concluem que perfis com mais informações sobre os anfitriões são julgados mais confiáveis do que perfis com menos informações. Uma categorização feita pelos autores até mostra quais categorias de informações disponibilizadas nos perfis são melhores indicadores de confiança.

As pesquisas sobre confiança na economia colaborativa têm mostrado os aspectos que podem influenciar na confiabilidade dos sistemas (e.g., reputação dos ofertantes, introdução de legislações coerentes, frameworks capazes de medir e melhorar a confiança, o papel das informações pessoais). Esses trabalhos trataram os aspectos relacionados à confiança individualmente, ou seja, cada artigo foca em um aspecto de confiança distinto e utilizando, na maioria dos casos, apenas um sistema como foco. Assim, nosso trabalho tem por objetivo ampliar este cenário, contemplando os principais aspectos de confiança que vêm sendo estudados por pesquisadores em sistemas de economia colaborativa.

\section{Metodologia}

Para investigar as questões de pesquisa relacionadas à confiança na economia colaborativa, uma Revisão Sistemática da Literatura (RSL) foi conduzida nas bases de dados da computação. A RSL, como foi definida por [Kitchenham 2004], é um meio de identificar, avaliar e interpretar todos os trabalhos relacionados à uma determinada questão de pesquisa, os tópicos de uma área do conhecimento, ou um fenômeno de interesse que deseja-se explorar. Neste trabalho, a RSL foi utilizada para coletar e analisar os trabalhos que apresentam pesquisas sobre economia colaborativa e seus processos de confiança.

A RSL foi realizada entre os meses de janeiro e março de 2017. Como estamos tratando de um tema recente, não excluímos nenhum trabalho baseado no período de publicação. O primeiro autor realizou a leitura completa e o registro dos dados de todos os artigos, e sua análise foi discutida com a segunda autora. A busca foi feita nos principais repositórios de artigos da área de computação: $\mathrm{ACM}^{3}$, $\mathrm{IEEE}^{4}$ e $\mathrm{ScienceDirect}^{5}$. A busca focou em artigos no idioma inglês. A string desenvolvida foi a seguinte:

(trust OR confidence OR reliability) AND ("sharing economy" OR "collaborative consumption" OR "collaborative economy" OR "co-production")

Apesar da string de busca ter sido desenvolvida focando no idioma inglês, isso não impediu que uma versão traduzida da busca tenha sido testada na língua portuguesa, todavia, nenhum resultado foi retornado nas buscas. Era de conhecimento dos autores que apenas algumas conferências do Brasil são indexadas por essas bases internacionais

\footnotetext{
${ }^{3}$ http://dl.acm.org/

${ }^{4} \mathrm{http}: / /$ ieeexplore.ieee.org/Xplore/home.jsp

${ }^{5} \mathrm{http}: / /$ www.sciencedirect.com/
} 
(e.g., IHC e Webmedia). A string proposta considerou os sinônimos de confiança do idioma inglês (i.e., trust, confidence, e reliability), que foram três termos encontrados em trabalhos que tratavam do assunto, e os quatro termos que os autores da área vêm comumente aplicando ao fenômeno de economia colaborativa (i.e., sharing economy, collaborative consumption, collaborative economy, e co-production), como mostrado por [Lampinen et al. 2015]. Na aplicação da RSL, testes foram realizados acrescentando os principais sistemas de economia colaborativa (e.g., Airbnb, Uber, dentre outros) na string para verificar se mais artigos seriam encontrados. Apesar de mais artigos terem sido encontrados pela string, uma leitura dos títulos e resumos, e em alguns casos, leitura diagonal dos mesmos, revelou que os artigos não faziam parte do tema de interesse, portanto, a string de busca é independente dos sistemas/aplicações de economia colaborativa.

A partir dos artigos retornados pela string de busca, os seguintes passos seriam executados para extração das informações relevantes das pesquisas: (1) leitura do título e resumo de todos artigos retornados, (2) leitura diagonal dos artigos retornados, e (3) leitura completa dos artigos retornados. No total, foram retornados 103 artigos considerando-se os 3 repositórios. Esta quantidade de artigos parece pequena para qualquer contexto da computação, todavia, a economia colaborativa foi definida em meados de 2010 [Botsman and Rogers 2010], e apenas em 2012 este fenômeno começou a se estabelecer ao redor do mundo [Lampinen et al. 2015]. Com isso, a maioria dos trabalhos retornados são de 2015 e 2016, sendo que o artigo mais antigo encontrado foi publicado em 2013. Isso mostra, o interesse recente de pesquisas sobre o assunto, sobretudo em função da popularidade que algumas plataformas tem adquirido recentemente (e.g., Uber e Airbnb). Ao final das etapas da RSL para seleção dos artigos, obteve-se 22 artigos relevantes para a questão de pesquisa definida, confrorme mostrado na Tabela 1.

Tabela 1. Resultado da String de Busca

\begin{tabular}{|l|c|c|c|}
\hline \multicolumn{5}{|c|}{ Revisão Sistemática da Literatura } \\
\hline Base de Dados & Título/Resumo & Leitura Diagonal & Leitura Completa \\
\hline ACM & 76 & 46 & 15 \\
\hline IEEE & 6 & 2 & 1 \\
\hline ScienceDirect & 21 & 12 & 6 \\
\hline Total & 103 & 60 & 22 \\
\hline
\end{tabular}

Outro fator importante de uma RSL são os critérios de inclusão de artigos que podem ter ficado de fora da busca. Como critério de inclusão, a string de busca foi aplicada na base do Google Scholar ${ }^{6}$. Essa busca priorizou artigos que ainda não foram publicados, e.g., a string encontrou um artigo relevante do CSCW'17, e também publicações de conferências independentes que não são anexadas por nenhuma das três bases descritas anteriormente. A busca no Google Scholar descartou os artigos que não fossem de conferências ou teses/dissertações. Assim, três artigos daquela base foram adicionados para análise completa, resultando assim em 25 artigos. Como critério de exclusão, artigos curtos de painéis de conferências foram excluídos da RSL.

Para cada artigo lido registramos várias informações que estariam relacionadas com a confiança na economia colaborativa. Dentre as informações registradas estão: (1)

\footnotetext{
${ }^{6}$ https://scholar.google.com.br/
} 
definição de confiança para os autores, (2) indicadores de confiança estudados, (3) tipos de métodos utilizados para analisar confiança, (4) sistema usado como estudo de caso, (5) tipos de valores troca indicados, (6) objetivos e contribuições importantes e (7) trabalhos futuros. Outras informações menos relevantes também foram coletadas na RSL. Para considerar a relevância do artigo, após sua leitura completa, foram definidos os seguintes critérios de qualidade: (1) definição e tratamento da confiança no artigo; (2) qualidade do método aplicado no contexto; e (3) contribuições e trabalhos futuros interessantes. De acordo com esses critérios foram eliminados 4 da lista final, após sua leitura completa, por não terem alcançado o nível de relevância desejado para a pesquisa.

\section{Resultados}

Essa seção apresenta os resultados dessa pesquisa. Primeiramente, fazemos uma descrição dos sistemas utilizados nas análises dos trabalhos estudados. Embora a identificação destes sistemas não fosse um objetivo direto da nossa pesquisa, ela é útil para termos uma visão geral dos sistemas sendo explorados. Além disso, a subseção facilitará o entendimento de alguns pontos utilizados para explicar os resultados. Nas três subseções que se seguem descrevemos os resultados obtidos para cada uma das subquestões de pesquisa descritas na introdução (QP.1, QP.2 e QP.3).

\subsection{Sistemas Usados na Economia Colaborativa}

As plataformas mais populares no contexto atual são o Uber e o Airbnb [Oh and Moon 2016]. O Uber é um aplicativo que permite a um ofertante usar seu veículo particular para oferecer deslocamentos para demandantes de uma origem a um destino. O serviço é similar aos serviços de táxis tradicionais, no entanto, os preços dos deslocamentos ofertados pelo Uber são muito menores, e por isso, têm atraído diversos usuários ao redor do mundo [Pargman et al. 2016]. Isso cria um ciclo que beneficia tanto o ofertante como o demandante do sistema, uma vez que o ofertante recebe o pagamento pelo deslocamento, enquanto o demandante usufrui do serviço a um custo menor que outros serviços convencionais de deslocamento.

O Airbnb é um sistema online (com versões web e para celular) que permite a ofertantes colocarem suas residências disponíveis para demandantes alugarem por um determinado período de tempo com determinadas regras e políticas de uso [Ikkala and Lampinen 2014, Lee et al. 2015, Fradkin et al. 2015]. Esta plataforma vem desafiando a indústria hoteleira ao redor do mundo, sobretudo, em função dos preços mais acessíveis que acomodações tradicionais [Lampinen and Cheshire 2016].

Em uma abordagem similar ao Airbnb, o sistema de economia colaborativa Couchsurfing ${ }^{7}$ oferece aos participantes a oportunidade de colocar sua residência à disposição de outros usuários que desejam acomodar-se durante um determinado período de tempo [Jung et al. 2016]. Diferente do Airbnb, no Couchsurfing o demandante não paga pela estadia, e a motivação para o ofertante é social (e não financeira).

O TaskRabbit ${ }^{8}$, por sua vez, provê meios para que os seus usuários ofereçam entre si os seus serviços para a realização de tarefas domésticas, tais como: fazer a compra do supermercado, mudanças, faxinas e pequenos reparos em residências

\footnotetext{
${ }^{7}$ https://www.couchsurfing.com/

${ }^{8} \mathrm{http}: / /$ www.taskrabbit.com
} 
[Dillahunt and Malone 2015]. O sistema permite que um demandante cadastre uma tarefa, e um ofertante realize a tarefa por um valor/hora pelo serviço. A plataforma tenta manter a qualidade do serviço por meio de revisões e outros mecanismos de avaliação de ofertantes e demandantes.

Os artigos também citam também plataformas criadas para que pessoas emprestem objetos que estão disponíveis para utilização de outros usuários do sistema, como o NeighborGoods ${ }^{9}$ e o Peerby ${ }^{10}$ [McLachlan et al. 2016]. Nestes sistemas, uma pessoa pode disponibilizar uma furadeira para empréstimo de outras pessoas, por exemplo. Além disso, o relacionamento é recíproco entre os participantes, tendo em vista que o usuário que emprestou o objeto tem o direito de pegar um objeto emprestado que esteja precisando [Dillahunt and Malone 2015].

É importante ressaltar que os sistemas que lideram este novo tipo de mercado, Airbnb e Uber, são diferentes do conceito original proposto pela economia colaborativa[Pargman et al. 2016]. A definição original previa que sistemas de economia colaborativa realizariam trocas de serviços sem haver uma troca monetária por esses serviços. Entretanto, pesquisadores têm julgado evidente que muitos desses sistemas visam somente maximizar seus lucros, fazendo da troca monetária o principal insumo dos sistemas, tornando as plataformas apenas provedores de serviços online-to-offline [Oh and Moon 2016]. Sendo assim, os sistemas deixam de apenas incentivar o compartilhamento de recursos ociosos de seus participantes, mas focam no aumento do lucro das plataformas.

\subsection{QP.1 Indicadores de Confiança}

A etapa de análise dos artigos da RSL nos permitiu identificar que indicadores estavam sendo utilizados para analisar ou investigar a confiança em sistemas de economia colaborativa. A partir desta análise, propomos um modelo no qual organizamos os indicadores utilizados na literatura de acordo com a dimensão a que se refere: sistema, pessoa (ou usuário) ou serviço. O modelo é independente de sistema e participantes, ou seja, não é focado em nenhuma ferramenta específica da economia colaborativa, nem mesmo no perfil do usuário (i.e., ofertantes ou demandantes). O Sistema é a plataforma que os usuários acessam para participar da economia colaborativa, por exemplo: o aplicativo do Uber nos smartphones, o site ou aplicativo do Airbnb, o site do TaskRabbit, entre outros. A dimensão Pessoa é formada pelos usuários desses serviços, sejam eles demandantes ou ofertantes [Botsman and Rogers 2010]. E a dimensão Serviço é constituída pelo serviço sendo oferecido pelo ofertante ao demandante através do sistema.

Para cada dimensão foram identificados os indicadores de confiança associados a ela. Assim como as dimensões, os indicadores propostos são baseados nos indicadores identificados na literatura analisada. Alguns indicadores propostos representam um indicador identificado em um conjunto de artigos, este por exemplo é o caso do indicador Qualidade que é usado em [Ert et al. 2016, Fradkin et al. 2015]. Por outro lado, outros indicadores foram gerados a partir do agrupamento temático de indicadores similares. Por exemplo, o indicador Satisfação agrupa os indicadores 'Competência e Satisfação' apresentado em [Ma et al. 2017] e 'Revisões e Comentários' apresentado

\footnotetext{
${ }^{9} \mathrm{http}: / /$ neighborgoods.net/

${ }^{10}$ https://www.peerby.com/
} 
em [Ert et al. 2016, Pick 2012]. 'Competência e Satisfação' é específico para o contexto do Airbnb devido às características daquela plataforma, enquanto que 'Revisões e Comentários' se refere às avaliações que usuários são capazes de prover sobre o serviço de ofertantes na economia colaborativa. Como ambos tratam de avaliação da satisfação com o serviço ofertado, então os agrupamos sob um mesmo indicador mais geral Satisfação. A seguir apresentamos cada um dos indicadores associado às dimensões. A Tabela 2 mostra os artigos que fundamentaram os indicadores, permitindo a rastreabilidade da análise.

Tabela 2. Rastreabilidade do Modelo de Confiança

\begin{tabular}{|l|l|l|l|}
\hline \multicolumn{4}{|c|}{ Rastreabilidade Modelo } \\
\hline Indicador & Referência & Indicador & Referência \\
\hline Segurança Perc. & [Dillahunt and Malone 2015] & Privacidade & [Dillahunt and Malone 2015] \\
\hline Risco & [Mohlmann 2016] & Conteúdo & {$[$ Ma et al. 2017] } \\
\hline Familiaridade & {$[$ Bilgihan et al. 2015] } & Qualidade & {$[$ Ert et al. 2016, Fradkin 2015] } \\
\hline Dados & [Oh and Moon 2016] & Satisfação & [Ert et al. 2016, Ma et al. 2017, Pick 2012] \\
\hline Autenticidade & [Pick 2012] & Compensação & [Han et al. 2016] \\
\hline
\end{tabular}

A Figura 1 mostra os indicadores associados a cada dimensão. Três indicadores foram identificados como estando associados à dimensão Sistema: Segurança Percebida, Risco e Familiaridade. A Segurança Percebida é uma característica da plataforma, e está relacionada com a segurança que o sistema de informação deve oferecer para as pessoas. O Risco é uma característica da plataforma relacionada ao risco que utilizar o sistema de economia colaborativa pode trazer aos seus usuários. Finalmente, a Familiaridade está relacionada à facilidade com que usuários aprendem a usar a plataforma.

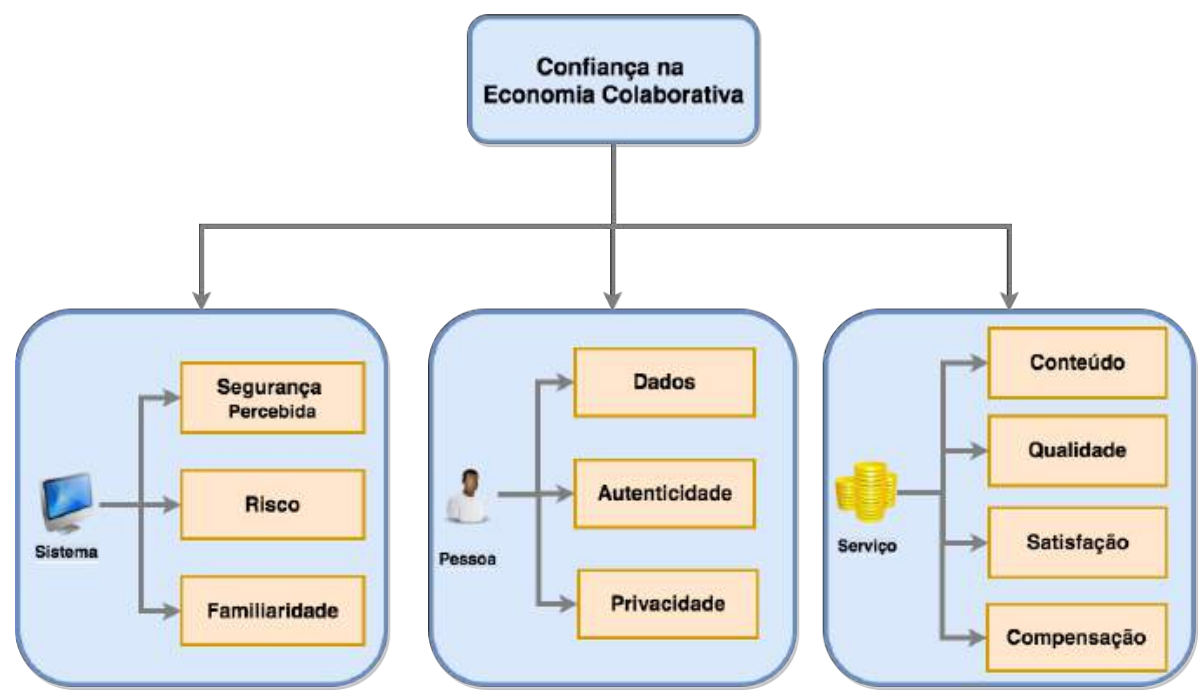

Figura 1. Modelo de Confiança

À dimensão Pessoa, foram associados 3 indicadores: Dados, Autenticidade e Privacidade. Os Dados de uma pessoa estão relacionadas às informações pessoais que ofertantes/demandantes disponibilizam nos sistemas de economia colaborativa. Autenticidade, por sua vez, está relacionada com a autenticação pelos usuários de suas informações junto aos sistemas. Por exemplo, o Airbnb permite que o usuário envie um documento 
(e.g. identidade) para comprovar os dados informados. A Privacidade é a medida do nível de privacidade atribuído pelo usuário ao uso do sistema de economia colaborativa.

Finalmente, em relação ao Serviço foram identificados 4 indicadores: Conteúdo, Qualidade, Satisfação e Compensação. Conteúdo representa as informações relacionadas ao serviço que os ofertantes fornecem, por exemplo, o serviço de transporte no Uber ou o empréstimo do quarto no Airbnb. A Qualidade está relacionada com a avaliação do serviço feita pelos usuários envolvidos tanto na prestação deste serviço, quanto na sua recepção. Qualidade pode ser um indicador tanto qualitativo, por exemplo no caso de uma avaliação na forma de comentário sobre o serviço ofertado, quanto quantitativo, por exemplo uma nota de 1 a 5 em um ou mais critérios de avaliação (no Airbnb, o freguês avalia a limpeza do local, sua localização, dentre outros). A Satisfação indica o quão satisfeito os usuários ficaram com a prestação do serviço [Ert et al. 2016, Ma et al. 2017, Pick 2012], tanto do lado do ofertante, quanto do demandante. Por exemplo, no CouchSurfing, o hóspede pode avaliar se ele se sentiu satisfeito com o anfitrião e o indicaria para outros hóspedes da rede. É importante ressaltar que os conceitos de qualidade e satisfação podem variar dependendo do sistema analisado, e.g., alguns sistemas permitem que demandante e ofertante se avaliem mutualmente, como no Airbnb, mas outros permitem a avaliação do demandante para o ofertante, como no TaskRabbit. A Compensação são as ações tomadas pelos sistemas em relação ao serviço em caso de qualquer problema que os usuários vivenciem durante a interação com o sistema de economia colaborativa, por exemplo, caso o hóspede decida cancelar uma reserva no Airbnb, quais são os meios de compensação que a plataforma fornece aos usuários.

Este modelo de confiança, apesar de estar na sua concepção inicial, já contribui para pesquisadores e desenvolvedores da área, pois apresenta uma visão geral dos aspectos sendo considerados relevantes para oferecer confiabilidade nos sistemas de economia colaborativa. Assim, ele pode servir de base para guiar o projeto ou análise destes sistemas, servindo de base para a reflexão e discussão sobre indicadores de confiabilidade.

A próxima seção descreve os métodos de pesquisa que têm sido utilizados em trabalhos que lidem com confiança na economia colaborativa. A descoberta dos métodos de pesquisa que estão sendo usados, ajuda a decidir quais métodos serão utilizados para validar este modelo futuramente.

\subsection{QP.2 Métodos Utilizados}

A partir da RSL, foi possível saber quais métodos de pesquisa qualitativos ou quantitativos têm sido utilizados em trabalhos que focam na confiança na economia colaborativa. A Tabela 3 apresenta uma visão geral dos métodos usados e com que frequência. Pode-se perceber que os métodos qualitativos de pesquisa foram os mais aplicados no contexto de confiança (44\%), enquanto que os métodos quantitativos foram um pouco menos aplicados (32\%). A combinação de ambos os métodos foi utilizada em (16\%) dos artigos, e (8\%) dos artigos não usaram nenhum tipo de método. Os artigos que não usaram métodos contemplaram pesquisas de análises da evolução de sistemas de economia colaborativa.

Os métodos qualitativos, em sua maioria, foram conduzidos por meio de entrevistas semi-estruturadas com participantes de sistemas de economia colaborativa [Pick 2012, Ikkala and Lampinen 2014, Jung et al. 2016, Lampinen and Cheshire 2016], sendo que esse instrumento de pesquisa foi pouco combinado com outros métodos quando 
Tabela 3. Métodos Utilizados na Literatura

\begin{tabular}{|l|l|l|l|}
\hline Tipo & $\mathbf{( \% )}$ & Descrição & Artigos \\
\hline Quali. & $44 \%$ & $\begin{array}{l}\text { Entrev. semi-estrut., } \\
\text { observ. e questioná. }\end{array}$ & $\begin{array}{l}\text { [Lumeau 2015, Santosoa 2015, Pick 2012, Oh and Moon 2016, } \\
\text { Dillahunt and Malone 2015, Ikkala and Lampinen 2014, } \\
\text { Lampinen and Cheshire 2016, Pargman et al. 2016] }\end{array}$ \\
\hline Quant. & $32 \%$ & $\begin{array}{l}\text { Coleta de dados e } \\
\text { question. fechados }\end{array}$ & $\begin{array}{l}\text { [Sanchez et al. 2016, Bilgihan et al. 2015, } \\
\text { Nunes and Correia 2013, Han et al. 2016, } \\
\text { Lee et al. 2015, Fradkin et al. 2015] }\end{array}$ \\
\hline Mistos & $16 \%$ & $\begin{array}{l}\text { Entrev. + Coleta } \\
\text { dados automáticas }\end{array}$ & [Ert et al. 2016, Hartl et al. 2016, Ma et al. 2017, Jung et al. 2016] \\
\hline NA & $8 \%$ & $\begin{array}{l}\text { Revisões trabalhos } \\
\text { economia colabor. }\end{array}$ & [Lampinen and Cheshire 2016] \\
\hline
\end{tabular}

aplicado. Além de entrevistas, os métodos utilizados foram observações de uso dos sistemas de economia colaborativa durante todo o processo de execução de uma tarefa [Ert et al. 2016, Pick 2012, Lumeau et al. 2015] e coleta de dados por meio de questionários com questões abertas [Dillahunt and Malone 2015]. Em relação aos métodos quantitativos, os autores optaram, na maioria dos casos, por questionários online com usuários de plataformas de economia colaborativa [Hartl et al. 2016, Bilgihan et al. 2015, Nunes and Correia 2013] ou por coletas automáticas nos sistemas de economia colaborativa mais conhecidos (e.g., Airbnb [Ert et al. 2016, Fradkin et al. 2015, Lee et al. 2015]).

Os artigos que adotaram métodos mistos, em geral utilizaram algum tipo de coleta de dados (i.e., coleta automática ou questionários) em uma das plataformas de economia colaborativa, em combinação com uma avaliação qualitativa envolvendo usuários para categorização de algum aspecto relacionado a confiança por meio da interpretação das informações contidas nos perfis dos participantes. Este foi o caso da análise do papel das fotos pessoais na confiança dos usuários do Airbnb [Ert et al. 2016]; da influência das informações no perfil do ofertante na confiança dos demandantes [Ma et al. 2017]; e das motivações dos usuários em utilizar os sistemas de economia colaborativa [Jung et al. 2016]. Além disso, foi utilizada a combinação de questionários com questões abertas e fechadas para um melhor entendimento do processo de possessão em economia colaborativa [Hartl et al. 2016].

De uma forma geral, métodos qualitativos são mais utilizados para entender um novo contexto, ou as motivações e razões das pessoas para usar uma tecnologia, ou mesmo o impacto da tecnologia na sociedade. Sendo assim, isso pode indicar que a maior quantidade de artigos que tenham usado métodos qualitativos em vez de quantitativos, seja devido ao fato da economia colaborativa ser muito recente [Botsman and Rogers 2010], onde pesquisadores ainda estão tentando entender por completo o fenômeno de colaboração entre as pessoas usando a tecnologia como ferramenta de interação entre participantes.

Os trabalhos que usaram métodos quantitativos, de uma forma geral, optaram por caracterizações do uso que os participantes têm feito da economia colaborativa [Sánchez et al. 2016, Möhlmann 2016, Lee et al. 2015, Fradkin et al. 2015]. Em outras palavras, identificar indicadores e seus impactos nos participantes, e.g. saber como as 
fotos de perfis podem influenciar na confiança dos participantes. Em contrapartida, os resultados indicam que os métodos qualitativos mostram "porque" os participantes tem aderido a essa nova economia. Nesse sentido, os métodos qualitativos têm sido usados, na sua maioria, para entender o conceito de confiança e acrescentar novas questões à área.

\subsection{QP.3 Valores Trocados}

Outra questão de interesse que analisamos através da RSL foram os valores trocados nos sistemas de economia colaborativa. Identificamos quatro valores trocados abordados pelos artigos: monetário, social, reputação e objetos. O valor monetário é aquele quando demandantes usam dinheiro para pagar pelo serviço de um ofertante (e.g., pagar por uma corrida do Uber). O valor social existe quando a interação social é a razão de utilização de um determinado sistema de economia colaborativa (e.g., Couchsurfing permite que ofertantes hospedem demandantes sem previsão de troca de valor monetário entre eles). A reputação é um valor negociado quando o serviço é uma avaliação dos envolvidos (e.g., o WhyTrusted ${ }^{11}$ permitia a troca de avaliações). E finalmente, o valor de objetos ocorre quando um sistema permite aos ofertantes emprestarem, por um determinado período de tempo, objetos que demandantes necessitem (e.g., como ocorre no NeighborGoods).

Vale ressaltar que um sistema de economia colaborativa pode permitir a troca de um ou mais destes valores. Na nossa análise, identificamos quais valores eram trocados nos sistemas abordados nos artigos e classificamos a importância deste valor no sistema. Por exemplo, dada uma plataforma como o Airbnb ou Uber (as duas plataformas mais presentes nas pesquisas), a troca monetária é considerada a troca primária entre os participantes [Jung et al. 2016, Lee et al. 2015, Lampinen and Cheshire 2016], enquanto que a troca social seria considerada a troca secundária naqueles dois sistemas. Caso o artigo apresentasse sistemas diferentes em termos de tipos de troca (e.g., Airbnb e NeighborGood), foi considerado o tipo de troca do sistema que era tema central do artigo, em seguida, o valor do sistema que não era o foco como secundário. É importante ressaltar que nesta análise o tipo de troca está relacionado ao artigo, e não ao sistema de economia colaborativa. A classificação considerou o entendimento que os autores dos artigos atribuíram aos sistemas em questão, e não exatamente ao senso comum que a comunidade possa ter em relação a um determinado sistema de economia colaborativa. A Tabela 4 mostra que valores foram abordados em que artigos e para que sistemas.

Os dados coletados mostram que a troca de valores monetários foi a troca mais discutida nos artigos abordados. Em segundo lugar aparece a troca social, seguida pela troca de reputação e objetos. A predominância da análise de sistemas que se baseiam em troca monetária pode ser um indicador da maior popularidade destes. A maior popularidade destes sistemas pode indicar uma evolução dos sistemas de economia colaborativa em relação à sua definição originalmente proposta por [Botsman and Rogers 2010], que discutia o caráter não monetário da economia colaborativa na interação dos seus participantes. Além disso, esse resultado indica que os sistemas de troca monetária foram o de maior interesse pelos pesquisadores que estudam os processos de confiança nesse novo tipo de economia. Este interesse pode estar relacionado a vários fatores distintos, por exemplo podem ter sido selecionados por sua popularidade (inclusive internacional),

\footnotetext{
${ }^{11} \mathrm{O}$ WhyTrusted é um sistema extinto, que permitia aos participantes avaliarem serviços prestados na economia colaborativa.
} 
Tabela 4. Valores trocados

\begin{tabular}{|c|c|c|c|}
\hline Valores & Primário & Secundário & Terciário \\
\hline Monetário & $\begin{array}{l}\mathbf{1 4} \text { artigos [Ert et al. 2016, Sanchez } \\
\text { et al. 2016, Hartl et al. 2016, } \\
\text { Bilgihan et al. 2015, Han et al. 2016, } \\
\text { Mohlmann 2016, Ma et al. 2017, } \\
\text { Pargman et al. 2016, Lee et al. 2015, } \\
\text { Fradkin et al. 2015]; 5 Sistemas } \\
\text { (Airbnb, ShareNow, Uber, } \\
\text { Sistema Autores, Lyft) }\end{array}$ & $\begin{array}{l}\mathbf{2} \text { artigos [Ikkala and Lampinen } 2014 \text {, } \\
\text { Jung et al. 2016]; } \\
1 \text { Sistema (Airbnb) }\end{array}$ & $\begin{array}{l}\mathbf{1} \text { artigo } \\
\text { [Dillahunt } \\
\text { and } \\
\text { Malone } \\
2015 \text { ] }\end{array}$ \\
\hline Social & $\begin{array}{l}3 \text { artigos [Dillahunt and Malone } \\
\text { 2015, Ikkala and Lampinen 2014]; } \\
\mathbf{2} \text { Sistemas (CouchSurfing, } \\
\text { TaskRabbit) }\end{array}$ & $\begin{array}{l}\mathbf{1 4} \text { artigos [Ert et al. 2016, } \\
\text { Sanchez et al. 2016, Hartl et al. 2016, } \\
\text { Bilgihan et al. 2015, Han et al. 2016, } \\
\text { Ma et al. 2017, Mohlmann 2016, } \\
\text { Pargman et al. 2016, Lee et al. 2015]; } \\
\mathbf{2} \text { Sistemas (Airbnb, Uber) }\end{array}$ & \\
\hline Reputação & $\begin{array}{l}\mathbf{2} \text { artigos [Nunes and Correia 2013, } \\
\text { Pick 2012]; } 2 \text { Sistemas } \\
\text { (PeerBy, WhyTrusted) }\end{array}$ & & \\
\hline Objetos & $\begin{array}{l}1 \text { artigo [McLachlan et al. 2016]; } \\
1 \text { Sistema (NeighborGood) }\end{array}$ & $\begin{array}{l}1 \text { artigo [Dillahunt and Malone 2015]; } \\
1 \text { Sistema (NeighborGood) }\end{array}$ & \\
\hline
\end{tabular}

o que também teria como consequência um maior número de usuários, e logo, potenciais participantes para análises qualitativas e/ou quantitativas; ou ainda, para as pesquisas quantitativas a facilidade de coleta e análise dessas plataformas pode ser relevante.

No entanto, é importante ressaltar que embora um sistema possa ter um valor principal sendo trocado (e.g., Airbnb o principal valor trocado é o monetário), outros valores podem ser consideradas secundários. Existem situações onde os valores de troca secundários são mais importantes para os usuários. Isso foi mostrado por [Jung et al. 2016] que discute como a troca social no Airbnb era mais relevante para diversos usuários que a monetária, mas isso não muda o tipo de troca primordial daquele sistema.

\subsection{Confiança em Sistemas de Economia Colaborativa}

Como descrito na seção de Introdução deste trabalho de pesquisa, a RSL seria usada para responder a seguinte questão de pesquisa: $Q P$. "Qual a definição de confiança no contexto de economia colaborativa?". A partir da leitura e aprofundamento na área de confiabilidade na economia colaborativa, uma boa definição para confiança seria:

A confiança é multifacetada, usada para diminuir riscos e incertezas criando uma sensação de segurança dos participantes. A confiança é transmitida em dois sentidos, tanto do demandante para o ofertante, quanto do ofertante ao demandante, mas tem um papel importante mediado pela plataforma, e exige níveis mais altos do que em sistemas peer-to-peer tradicionais (e.g., E-bay), sobretudo, devido ao contato social e ao envolvimento de um serviço e não um produto na troca entre os participantes. Sistemas de economia colaborativa podem envolver a troca de diferentes tipos de valores. Esses valores podem influenciar aspectos de confiança. Por exemplo, a troca monetária ou de 
objetos podem priorizar diferentes aspectos da confiança.

Essa definição foi elaborada cuidadosamente com base na análise da RSL, e explica algumas das características da economia colaborativa. A confiança é multifacetada, pois depende de diversos indicadores, como mostrado no nosso modelo. Ela pode passar de um ofertante a um demandante, ou vice-versa, durante a troca de valores, entretanto, a plataforma tem um papel imprescindível na troca entre os participantes pois é o canal de comunicação entre os usuários [Möhlmann 2016]. Outros artigos até discutem que uma interface difícil de navegar e com baixa usabilidade não contribui para confiança no sistema [Bilgihan et al. 2015]. A economia colaborativa, diferentemente de outros tipos de relações peer-to-peer, prevê que na maioria dos casos será necessária uma interação social entre os usuários, o que por si só já é um motivo para que seja necessária uma confiança maior do que em outras interações online [Jung et al. 2016]. Com a crescente evolução desse tipo de economia, a troca monetária tem se tornado o principal tipo de troca entre os participantes [Oh and Moon 2016, Pargman et al. 2016]. Os sistemas de economia colaborativa podem afetar as interações sociais dos usuários como previsto por [Botsman and Rogers 2010], porém, o advento de plataformas com foco nas trocas monetárias afeta o mercado comum como um todo, trazendo as preocupações do mercado online para a economia colaborativa, e.g., autenticidade dos usuários, segurança e privacidade das informações cadastradas, dentre outros aspectos. Nota-se que os tipos de valores trocados nesses sistemas interferem diretamente na confiança esperada por seus usuários.

\section{Conclusões}

Este artigo apresentou uma análise de como aspectos relacionados à confiança têm sido tratados em pesquisas sobre sistemas de economia compartilhada. A pesquisa foi realizada através da aplicação de uma Revisão Sistemática da Literatura no contexto de economia colaborativa. Os trabalhos existentes sobre o assunto tratavam a confiança de uma forma específica, seja em uma plataforma isolada, ou em um contexto de análise que lida com um número limitado de aspectos de confiança. Essa pesquisa é uma contribuição a área de sistemas de economia colaborativa, tendo em vista o estudo amplo que foi realizado na tentativa de definir a confiança e entender suas características. A pesquisa nos revelou que a questão de confiança na economia colaborativa envolve diferentes indicadores associados a diferentes dimensões (sistema, pessoa e serviço), conforme representado no modelo (Figura 1). Além disso, a análise feita nos permitiu identificar e descrever tanto as diferentes metodologias sendo utilizadas em pesquisas relacionadas à caracterização de confiança nestes, quanto os valores sendo trocados neste modelo econômico.

O modelo proposto é a representação visual de como a confiança tem sido tratada e expressa nos sistemas de economia colaborativa. Assim, traz uma contribuição para a pesquisa na área ao organizar e explicitar os elementos relevantes e suas relações. $\mathrm{O}$ passo seguinte nesta pesquisa envolve a avaliação deste modelo para que ele possa ser consolidado ou mesmo revisado. Além disso, nossa análise dos métodos sendo utilizados nas pesquisas mostrou que existem diversas possibilidades metodológicas para apreciar confiança na literatura, e que podem ser replicadas no modelo proposto. A própria concepção do modelo, no sentido de ser independente de plataforma ou tipo de usuário (i.e., demandante ou ofertante), facilita sua validação em outros contextos.

Em termos de contribuições, esse trabalho apresenta a identificação dos tipos de 
valores de troca, e também o levantamento do estado da arte na pesquisa sobre confiança em sistemas de economia colaborativa. Esses resultados podem ser de grande utilidade para pesquisadores trabalhando ou interessados em trabalhar na área.

Sobre as limitações desse trabalho, apesar de uma versão traduzida da busca ter sido aplicada nas bases da computação, pretendemos replicar a busca para as publicações da SBC. Esse processo foi dificultado uma vez que grande parte das conferências da SBC, dentre elas o SBSC, não estão indexadas em uma base que ofereça um mecanismo de busca (através de string de busca). Assim, pretendemos realizar uma busca manual nos eventos relevantes da SBC, mas que pelo seu custo foi deixado como um próximo passo. Além disso, na discussão sobre os tipos de valores de troca, os tipos apresentados na Tabela 4 podem não representar todos os praticados atualmente em sistemas de economia colaborativa. A metodologia proposta garantiu que as principais bases de dados da computação foram pesquisadas e analisadas na leitura dos artigos. No entanto, isso não impede que outros tipos de troca existam na literatura não investigada, ou mesmo, que existam tipos de troca que só acontecem na prática, i.e., no uso dos sistemas pelos usuários. Uma possível razão para o não aparecimento de determinado tipo de troca seria o foco da string de busca especificamente no conceito de confiança.

Finalmente, o modelo apresentado é resultado de uma análise interpretativa feita pelos autores. Assim, para permitir a avaliação do mesmo foi apresentado exemplos de dados que motivaram a interpretação, para permitir a avaliação da análise feita. No entanto, ainda não foi feita uma triangulação do modelo através da aplicação de outras pesquisas e análises. Este é um passo futuro necessário na direção da consolidação deste modelo.

\section{Agradecimentos}

Geanderson E. dos Santos agradece a FAPEMIG/Google pela sua bolsa de pesquisa.

\section{Referências}

Bilgihan, A., Nusair, K., Okumus, F., and Cobanoglu, C. (2015). Applying flow theory to booking experiences. Information Management.

Botsman, R. and Rogers, R. (2010). What's Mine is Yours: The Rise of Collaborative Consumption. HarperBusiness, New York, NY, USA.

Dillahunt, T. R. and Malone, A. R. (2015). In Proceedings of the 33rd Annual ACM Conference on Human Factors in Computing Systems, New York, NY, USA.

Ert, E., Fleischer, A., and Magen, N. (2016). Trust and reputation in the sharing economy: The role of personal photos in airbnb. Tourism Management, 55:62 - 73 .

Fradkin, A., Grewal, E., Holtz, D., and Pearson, M. (2015). Bias and reciprocity in online reviews: Evidence from field experiments on airbnb. In Proceedings of the Sixteenth ACM Conference on Economics and Computation, EC '15, New York, NY, USA.

Hartl, B., Hofmann, E., and Kirchler, E. (2016). Do we need rules for "what's mine is yours"? governance in collaborative consumption communities. Journal of Business.

Ikkala, T. and Lampinen, A. (2014). Defining the price of hospitality: Networked hospitality exchange via airbnb. In Proceedings of the Companion Publication of the 17th ACM Conference on Computer Supported Cooperative Work \&\#38; Social Computing, CSCW Companion '14, pages 173-176, New York, NY, USA. ACM. 
Jung, J., Yoon, S., Kim, S., Park, S., Lee, K.-P., and Lee, U. (2016). Social or financial goals?: Comparative analysis of user behaviors in couchsurfing and airbnb. In Proceedings of the 2016 CHI Conference Extended Abstracts on Human Factors in Computing Systems, CHI EA '16, pages 2857-2863, New York, NY, USA. ACM.

Kitchenham, B. (2004). Procedures for performing systematic reviews. Keele University Technical Report TR/SE-0401.

Lampinen, A., Bellotti, V., Monroy-Hernández, A., Cheshire, C., and Samuel, A. (2015). Studying the "sharing economy": Perspectives to peer-to-peer exchange. In Proceedings of the 18th ACM Conference Companion on Computer Supported Cooperative Work \&\#38; Social Computing, CSCW'15 Companion, New York, NY, USA.

Lampinen, A. and Cheshire, C. (2016). Hosting via airbnb: Motivations and financial assurances in monetized network hospitality. In Proceedings of the 2016 CHI Conference on Human Factors in Computing Systems, New York, NY, USA.

Lee, D., Hyun, W., Ryu, J., Lee, W. J., Rhee, W., and Suh, B. (2015). An analysis of social features associated with room sales of airbnb. In Proceedings of the 18th ACM Conference Companion on Computer Supported Cooperative Work \&\#38; Social Computing, CSCW'15 Companion, New York, NY, USA.

Lumeau, M., Masclet, D., and Penard, T. (2015). Reputation and social (dis)approval in feedback mechanisms: An experimental study. Journal of Economic Behavior, 112.

Ma, X., Hancock, J. T., Mingjie, K. L., and Naaman, M. (2017). Self-disclosure and perceived trustworthiness of airbnb host profiles. In Proceedings of the 20th ACM Conference on Computer Supported Cooperative Work and Social Computing Companion, CSCW'17, New York, NY, USA. ACM.

McLachlan, R., Opila, C., Shah, N., Sun, E., and Naaman, M. (2016). You can't always get what you want: Challenges in $\mathrm{p} 2 \mathrm{p}$ resource sharing. In Proceedings of the 2016 CHI Conference Extended Abstracts on Human Factors in Computing Systems, CHI EA '16, New York, NY, USA. ACM.

Möhlmann, M. (2016). Digital trust and peer-to-peer collaborative consumption platforms: A mediation analysis. New York University (NYU).

Nunes, M. and Correia, J. (2013). Improving trust using online credibility sources and social network quality in p2p marketplaces. In 2013 8th Iberian Conference on Information Systems and Technologies (CISTI), pages 1-4.

Oh, S. and Moon, J. Y. (2016). Calling for a shared understanding of the "sharing economy". In Proceedings of the 18th Annual International Conference on Electronic Commerce: E-Commerce in Smart Connected World, New York, NY, USA. ACM.

Pargman, D., Eriksson, E., and Friday, A. (2016). Limits to the sharing economy. In Proceedings of the Workshop on Computing Within Limits, New York, NY, USA.

Pick, F. (2012). Building trust in peer-to-peer marketplaces: An empirical analysis of trust systems for the sharing economy. B.S. Thesis, Zeppelin University.

Sánchez, D., Martínez, S., and Domingo-Ferrer, J. (2016). Co-utile $\{$ P2P $\}$ ridesharing via decentralization and reputation management. Transportation Research Part C: Emerging Technologies. 